\title{
RAPPORT D'ACTIVITÉ DE LA COMMISSION INTERNATIONALE DE BOTANIQUE APICOLE DE L'U.I.S.B. POUR LES ANNÉES 1966-1969
}

\author{
Anna MAURIZIO et Jean LOUVEAUX \\ 3097 Liebefeld Bern, Rosenweg 9 (Suisse) \\ Station de Recherches sur l'Abeille et les Insectes sociaux, \\ 91 - Bures-sur-Yvette \\ Institut national de la Recherche agronomique
}

Le présent rapport concerne l'activité de la Commission internationale de Botanique apicole de l'U.I.S.B. et de ses groupes de travail au cours des quatre dernières années, c'est-à-dire de la période comprise entre les Assemblées générales de Bucarest (1965) et de Münich (1969). Étant donné le nombre relativement peu important des membres ayant pu participer au Congrès international d'apiculture de Maryland on a du renoncer à organiser une Assemblée générale de la Com. mission à cette occasion; seul, le groupe de travail "Pollinisation " a pu tenir une réunion.

La Commission internationale de Botanique apicole de l'U.I.S.B. (C.I.B.A.) compte actuellement 175 membres appartenant à 34 pays. Au cours de la période que concerne le présent rapport elle a perdu par décès cinq de ses membres : N. M. GlushKov (U.R.S.S.), J. I. Hambleton(U.S.A.), B. Hazslinsky (Hongrie), W. Ludr (Suisse) et E. Wohlgemuth (R.F.A.). Dix-sept membres ont volontairement quitté la Commission cependant que vingt-sept adhésion nouvelles étaient enregistrées. Trois nouveaux pays se trouvent ainsi représentés : l'Angola, la République Argentine et la Belgique.

Vingt-quatre membres appartenant à 15 pays ont participé le 31 juillet 1969 à l'Assemblée générale qui s'est tenue à Münich. Différentes questions administratives ont été examinées parmi lesquelles une proposition de réorganisation de la Commission. Un vœu a été émis pour que l'organisation interne, jusqu'ici très libre, soit à l'avenir plus structurée. Dans ce but, le bureau a présenté à l'Assemblée générale un projet de réorganisation. Après discussion, la résolution suivante a été adoptée :

a. J. Louveaux est chargé de préparer pour la C.I.B.A. un projet de statuts et de le mettre au point en accord avec le secrétariat général de 1'U.I.S.B. et avec un comité. Le projet sera ensuite soumis par écrit à tous les membres de la C.I.B.A. et présenté pour discussion et adoption à la prochaine Assemblée générale.

b. Le bureau s'adjoint un trésorier en la personne de G. VoRwoHL.

c. Un conseil est élu.

$d$. A partir du $1^{\mathrm{er}}$ janvier 1970 il sera perçu une cotisation annuelle destinée à couvrir les menues dépenses de la Commission. Provisoirement, la cotisation ne sera perçue que sur les Instituts membres de la C.I.B.A.; son montant sera de 10 Dollars par an.

$e$. L'ordre du jour comportant l'examen de la proposition, déjà faite en 1965 par E.V. SANDULEAC, de créer un groupe de travail de "Biophysique et génétique ", l'Assemblée générale décide de reprendre cette proposition lorsque la réorganisation de la Commission sera achevée. 
Pendant la période que concerne le présent rapport, la Commission internationale de Botanique apicole a organisé ou a pris part aux réunions suivantes :

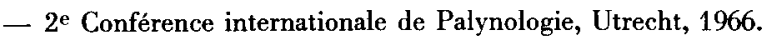

— réunion du groupe de travail "Recherches sur le miel " à Stuttgart-Hohenheim, 1967;

- comité des méthodes du Codex alimentarius europaeus, sessions de Berlin, 1967 et 1968;

- assemblée constitutive de l'Association des Palynologues de langue française, Paris, 1968;

- réunion restreinte relative aux questions de pollinisation et de protection des abeilles, Asker (Norvège), 1969;

- réunion commune du groupe de travail des Instituts de recherche apicole allemands et de la C.I.B.A. (Groupe de travail "Miellats") au Symposium scientifique du XXIII ${ }^{\mathrm{e}}$ Congrès international d'Apiculture, Münich, 1969;

- assemblée générale de la C.I.B.A., Münich, 1969.

Par ailleurs, la C.I.B.A. a publié ou contribué a la publication des documents suivants :

- rapport d'activité pour les années 1962 à 1965 (Bee World $46: 132,1965$. Ann. Abeille, 8: 325,1965 . Z. f. Bienenforschg. $8: 267,1966$ );

- proceedings of the 2nd International Symposium on Pollination (E. Akerberg \& E. Crane, Bee Research Association, Suppl. Bee World, vol. 47, 1966);

- "Pollens de plantes mellifères d'Europe" (A. Maunizio \& J. Louveaux, U.G.A.F., Paris, 1965);

- numéro spécial sur les Ericacées et leurs miels (Ann. Abeille, 9 : (4), 1966);

- numéro spécial consacré à la réunion de Stuttgart-Hohenheim du groupe de travail "Recherches sur le miel " (Z. f. Bienenforschg. 9 (5), 1968);

— circulaires $\mathrm{n}^{\text {os }} 22$ à 26 de la C.I.B.A., 1965-1969;

- listes $n^{05} 7$ (1965) et 8 (1969) des publications nouvelles intéressant le groupe de travail "Recherches sur le miel ";

- contributions diverses au "Traité de Biologie de l'Abeille" de R. Chauvin (Masson ed., Paris, 1968);

- contribution au "Waldhonigbuch" de W. Kloft, A. Maurizio \& W. Kaeser (Ehrenwirth ed., Münich, 1965);

- contribution au "Trachtpflanzenbuch » de A. Maurizio et I. Grafl (Ehrenwirth ed., Münich, 1969).

Mais l'activité de la Commission internationale de Botanique apicole est, avant tout, celle de ses groupes de travail. Les groupes "Recherches sur le miel ", "Miellats " et "Pollinisation " ont été particulièrement actifs. Les groupes de travail " Protection des abeilles » et "Flore méditerranéenne " sont en cours de réorganisation.

\section{1. - Groupe de travail "Recherches sur le miel"}

(Rapporteurs J. Louveaux et A. Maurizio)

La réunion qui s'est tenue à Stuttgart-Hohenheim les 11, 12 et 13 mai 1967 constitue sans dout : la manifestation d'activité la plus importante du groupe de travail "Recherches sur le miel " au cours de la période 1966-1969. Elle a permis de rassembler une trentaine de personnes appartenant à neuf pays différents et, pour la première fois, en dehors des spécialistes de l'analyse pollinique, on a noté la présence d'un nombre important de chimistes. Ainsi s'est trouvée pleinement concrètisée la volonté, manifestée depuis 1961, de donner au groupe de travail une orientation plus large permettant de couvrir l'ensemble des aspects scientifiques et techniques des recherches sur le miel. Quatorze communications ont été présentées. Elles ont été rassemblées dans le fascicule 5 du tome 9 de la Z. f. Bienenforschg. Le plus grand nombre d'entre elles concerne l'analyse pollinique mais on y trouve également plusieurs études purement chimiques ainsi que l'exposé de méthodes de caractérisation de miels monofloraux de production courante. Les problèmes de méthodologie et de terminologie ont également fait l'objet d'exposés et de discussions importantes.

En dehors de la réunion de Stuttgart-Hohenheim, il convient de noter que le groupe de travail était représenté, d'une part à la $2^{e}$ Conférence internationale de Palynologie d'Utrecht en 1966 et, d'autre part, à l'Assemblée constitutive do l'Association des Palynologues de langue française à Paris en 1968. Deux communications ont été présentées à Utrecht, essentiellement dans le but de manifester l'intérêt que notre Commission porte à la Palynologie en général. Bien que les méthodes 
de la mélisso-palynologie présentent un certain nombre de particularités qui en font une discipline trés spécialisée, les spécialistes du miel se doivent de conserver un contact étroit avec leurs collègues palynologues. Cette volontée de contacts avec l'ensemble des scientifiques qui étudient le pollen a conduit plusieurs d'entre nous à entrer dans l'Association des palynologues de langue française. L'entrée d'un membre du groupe de travail dans le comité international de Palynologie récemment créé montre, par ailleurs, la réciprocité du désir de voir s'établir des contacts étroits.

Le projet, depuis longtemps formé, de regrouper dans un document unique et facilement accessible à tous l'ensemble revu et mis à jour des méthodes de la mélisso-palynologie vient d'aboutir. Publiées en allemand, français et anglais, les "Méthodes de la mélisso-palynologie " viendront remplacer les « Méthodes de l'analyse pollinique des miels » diffusées par la C.I.B.A. en 1963. La nouvelle publication offrira l'avantage d'être beaucoup plus complète, de fixer de façon rationnelle la terminologie et de donner des indications précises sur le mode d'interprétation des résultats. Elle sert de document de base pour l'admission de la mélisso-palynologie dans les méthodes du Codex alimentarius europaeus et elle a été présentée au Comité des méthodes du Codex pour étude (Codex/Analys/68/10, 1968).

En conclusion, nous pouvons dire que les recherches sur le miel qui avaient semblé décliner il y a quelques années ont retrouvé récemment toute leur importance. La raison de ce regain d'intérêt est à rechercher sans doute dans le contexte économique actuel. Les producteurs, le négoce et les consommateurs exigent de plus en plus que les produits alimentaires, et parmi- eux le miel, répondent à des normes précises de qualité et offrent des garanties réelles d'origine. Les progrès considérables des méthodes d'anałyse donnent par ailleurs aux services officiels chargés du contrôle des denrées alimentaires des moyens nouveaux de vérifier la qualité et la loyauté des produits. Le groupe de travail "Recherches sur le miel " de la C.I.B.A. se doit de favoriser sur le plan international le progrès et la diffusion des connaissances scientifiques et techniques relatives au miel, qu'il s'agisse de sa composition ou de son analyse. Les listes de publications nouvelles régulièrement préparées et diffusées par la C.I.B.A. répondent à cet objectif.

\section{2. - Groupe de travail "Nectar"}

\section{(Rapporteurs R. W. Shuel et G. F. Townsend)}

L'activité du groupe de travail "Nectar " repose essentiellement sur l'échange d'informations et de données bibliographiques ainsi que sur des propositions pour de nouvelles recherches. Un rapport sur les recherches effectuées récemment en France, en Allemagne, en Israël, en Pologne, en Grande-Bretagne, aux Etats-Unis et au Canada ainsi que sur les tâches futures de la recherche sur le nectar paraîtra prochainement dans la revue "Bee-World".

D'un point de vue pratique les recherches poursuivies sur le nectar s'attaquent à deux problèmes : l'amélioration de la production du miel et l'augmentation de la production végétale grâce à une meilleure pollinisation. Pour atteindre le but ainsi fixé plusieurs méthodes sont possibles :

a. Modification du producteur de nectar par sélection ou action sur le milieu;

$b$. Sélection des espèces qui conviennent particulièrement bien aux différents biotopes;

c. Sélection des insectes pollinisateurs.

L'application de ces trois méthodes nécessite des recherches fondamentales. Les questions suivantes se trouvent aujourd'hui au premier plan de nos préoccupations :

\section{$1^{0}$ Influence de la température.}

Chez certaines espèces végétales il existe un seuil très net de température au-dessous duquel il ne se produit aucune secrétion nectarifère. Ceci laisse présager que la secrétion nectarifère dépend d'un ou de plusieurs systèmes enzymatiques. La question est de savoir quels sont ceux qui sont importants pour la secrétion nectarifère.

\section{$2^{\circ}$ Prévision de la miellée.}

Dans les régions où les forêts jouent un rôle important comme source de nectar il existe d'importantes variations annuelles dans l'intensité de la miellée qui, jusqu'ici, n'ont pas été clairement expliquées. La question qui se pose est de savoir si la miellée est influencée par les conditions qui ont régné au cours de l'année précédente. La miellée peut-elle être prévue? La prévision des miellées serait d'une très grande importance pour les territoires de transhumance dans certains pays (par ex. l'Australie). 


\section{$3^{0}$ Attraction des pollinisateurs.}

Quels sont les facteurs qui conditionnent l'attractivité des plantes pour les insectes? G. R. WYKES a montré que les proportions des principaux sucres présents dans le nectar exercent une certaine influence sur le choix que fait l'abeille entre les différentes sources de nourriture. Quel est le rôle joué parałlèlement par les arômes? Une étude comparative des variétés odorantes et non odorantes de la même espèce végétale pourrait apporter des informations à ce sujet.

\section{$4^{\circ}$ Facteurs hormonaux.}

Dans quelle mesure la sécrétion nectarifère est-elle dépendante de processus hormonaux? La sécrétion du nectar est-elle coordonnée avec le développement de la fleur, la maturation du pollen et la réceptivité du stigmate?

\section{3. - Groupe de travail "Miellats»}

(Rapporteur W. KLOFT)

Dans la période que couvre le présent rapport se place la publication, par des membres du groupe de travail, de plusieurs livres ou chapitres de livres d'une grande importance. Le travail en commun de W. Kaeser, A. Fossei, J. Schels, A. Maurizio et W. Kloft, a permis la réalisation du "Waldhonigbuch " (le livre du miel des forêts) édité par Ehrenwirth (1965) et qui constitue une tentative pour faire le point de nos connaissances actuelles tout en mettant en évidence les lacunes de celles-ci. Cette publication se trouve complétée et élargie par un livre de O. HaraGsim (1966) et par un chapitre de H. Muller dans l'ouvrage "Die Bienenweide" publié chez Ulmer (1967). Les contributions de A. Maunizıo et de W. KLort au "Traité de Biologie de l'Abeille " (Masson ed., 1968) ont permis d'étendre au domaine linguistique français une documentation qui lui faisait défaut. Les analyses bibliographiques se rapportant aux publications que nous venons de citer, y compris dans les périodiques de langue anglaise ou russe montrent le grand intérêt soulevé par les questions de miellats. On constate, dans l'ensemble, que : $1^{\circ}$ L'accroissement des connaissances fait que l'on reconnaît de plus en plus l'importance des miellées de miellat, non seulement en Europe, mais également en Asie, en Nouvelle-Zélande et en Amérique; $2^{\circ}$ L'emploi de plus en plus intensif des herbicides et des insecticides dans les pays possédant une agriculture évoluée oblige l'apiculture à compter davantage sur les miellats pour assurer son existence; $3^{\circ}$ Le développement constant des connaissances sur les agents de production des miellats et sur les miellées favorise la coopération entre la pratique, la science apicole et l'économie forestière ainsi que le montre la création de services de prévision des miellées, souvent avec l'appui des pouvoirs publics et des stations de radio, services qui ont déjà fait leurs preuves ou bien sont en cours de création.

Pour toutes ces réalisations les contacts entre collègues et le travail en commun des membres du groupe ont constitué l'ełément décisif des succès obtenus. Ils ont été grandement favorisés par la correspondance, le travaił d'équipe et les rencontres personnelles dans les réunions de spécialistes ou les congrès. $A$ ce point de vue le XIIIe Congrès international d'entomologie à Moscou fut très important. Une rencontre de spécialistes prévue à Halle à l'automne 1968 et à laquelle devaient participer des experts allemands, tchèques, hollandais et polonais n'a malheureusement pas pu avoir lieu. L'objectif principal reste maintenant de tirer au clair certains problèmes de taxonomie des Lachnides et principalement des espèces vivant sur les Pins. Le"groupe "de"travail devra."s'occuper de ces questions dans les années à venir.

Signalons enfin le"symposium sur les miellats qui a eu lieu à Münich"en 1969 et au cours"duquel 11 communications furent présentées, ainsi qu'un film. Les rapporteurs, membres du groupe de travail, appartenaient à 7 pays différents. Les communications présentées ont été vraiment le reflet de nos préoccupations actuelles et de l'activité du groupe de travail.

\section{4. - Groupe de travail « Protection des abeilles"}

\section{(Rapporteur W. STUTE)}

Les travaux de ce groupe intéressent deux domaines :

$1^{0}$ Examen des pesticides du point de vue de leur toxicité pour les abeilles;

$2^{\circ}$ Mise en évidence de ces mêmes substances dans les abeilles mortes. 
1. A l'intérieur du territoire de la République fédérale allemande on travaille dans les différentes stations compétentes (il en existe 10) selon des directives uniformes homologuées qui ont été publiées en 1969. Cette publication donne des instructions pour la détermination au laboratoire de l'action respiratoire, de contact et d'ingestion des pesticides ainsi que pour la mise en place d'essais sous cage ou en plein air et pour la présentation des résultats. Pour l'essentiel, ces méthodes recoupent celles qui sont recommandées depuis des années au sein du groupe de travail "Protection des abeilles". On a constaté en Allemagne que les résultats obtenus par les différentes stations chargées de procéder aux examens présentaient une très bonne concordance et restaient dans les limites de tolérance habituelles des expériences biologiques, de telle sorte que jusqu'ici, dans la plupart des cas, il a été possible de fournir sur une saison (de mai à septembre) une appréciation sur la toxicité relative des produits testés.

Dans la République fédérale allemande on continue à être d'avis qu'il convient de conserver la division des pesticides en deux catégories : dangereux pour les abeilles, non dangereux pour les abeilles. Cette division s'appuie sur le fait que sur le territoire fédéral on cultive des plantes très variées et, souvent, sur de petites surfaces; il en résulte des difficultés dans la surveillance des stations de traitement par les services officiels. Il est par ailleurs connu que dans certains pays on utilise trois ou même plus de trois catégories en ce qui concerne la toxicité pour les abeilles. Du fait que les circonstances locales conditionnent le mode de classement il ne paraît guère possible, dans ce domaine, d'aboutir à une normalisation internationale des catégories.

L'idée d'introduire dans les différents pays une dénomination unique pour les pesticides est accueillie favorablement et elle continue à être étudiée.

Par ailleurs, les membres du groupe de travail " Protection des abeilles " recevront ultérieurement un recueil des pesticides reconnus en Allemagne comme non dangereux pour les abeilles. On propose que des listes analogues établies dans les autres pays soient adressées au rapporteur pour permettre une coordination.

2. En ce qui concerne la mise en évidence des pesticides dans les abeilles mortes les travaux ne manquent pas dans les pays où le problème des intoxications est aigu. Les méthodes les plus diverses sont proposées. Le rapporteur serait très heureux si ces méthodes pouvaient lui être communiquées dans une forme abrégée de façon à ce qu'il puisse en présenter une synthèse aux membres du groupe de travail, tout comme en 1960.

De divers côtés est venue la suggestion d'unifier les groupes de travail qui existent dans notre secteur de recherches. Qu'il nous soit permis de remarquer que notre groupe de travail existe déjà depuis de nombreuses années et qu'il a apporté une contribution décisive aux travaux que nécessite une coopération internationale dans le domaine de la protection des abeilles.

Afin de ranimer cette coopération, le rapporteur lance ici même une invitation à tous ceux que la question intéresse pour l'année 1971 à Celle (R.F.A.).

\section{5. - Groupe de travail " Pollinisation"}

\section{(Rapporteurs E. Akerberg, G. Julen, N. Holm)}

Dans la période couverte par le présent rapport, le compte rendu du symposium sur la pollinisation tenu à Londres en 1964 a été publié (Proceeding of the 2nd International Symposium on Pollination, Bee Research Association, 1966).

En liaison avec le XXIe Congrès international d'apiculture à Maryland, 1967, a eu lieu une réunion pour les questions de pollinisation à laquelle 25 spécialistes ont pris part. Les question suivantes ont été discutées :

1. Introduction dans les "Apicultural abstracts » d'analyses de travaux sur la pollinisation;

2. Organisation du prochain symposium sur la pollinisation. La majorité des participants furent

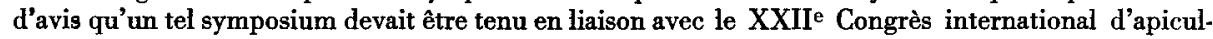
ture à Münich en 1969.

3. Discussion sur la pollinisation par les insectes de différentes plantes cultivées;

A la suite de cette réunion, il fut décidé qu'un symposium sur la pollinisation serait organisé en liaison avec le Congrès international d'apiculture de Münich. Une demande à ce sujet adressée au secrétariat du congrès reçut malheureusement une réponse trop tardive pour que le groupe de travail puisse se charger de l'organisation du symposium prévu. 
Un symposium limité aux pays scandinaves a eu lieu les 22 et 23 juillet 1969 à Asker en Norvège. A. MaUrizio, entre autres, y assistait. Un résumé des rapports présentés à cette réunion paraîtra prochainement dans le Bee World. Pendant la réunion eurent lieu des conversations en comité restreint comprenant principalement des représentants des pays nordiques. Il fut décidé que le groupe de travail "Pollinisation ", malgré l'importance qu'il a pris au cours des dernières années continuerait, comme par le passé, à travailler en liaison avec la C.I.B.A. L'éventualité d'une autonomie ne sera examinée qu'au prochain symposium.

Par ailleurs, le vœu a été émis de voir les "Apicultural abstracts" étendus aux recherches sur la pollinisation.

Le groupe de travail a reçu d'Australie une proposition pour organiser en 1972, en liaison avec le congrès international d'entomologie, un symposium sur la pollinisation. Cette proposition a été favorablement accueillie. D'autres informations à ce sujet seront publiées dans la revue "Bee World ".

\section{6. - Groupe de travail "Flore méditerranéenne" \\ (Rapporteurs J. LiNDER et J. RiHAR)}

Le groupe de travail "Flore méditerranéenne" une fois réorganisé, a présenté un programme de travail portant sur plusieurs années. Les recherches doivent commencer dans différents pays et être ensuite étendues à tout le bassin méditerranéen. Plusieurs laboratoires européens ont assuré le groupe de leur collaboration. Le programme de travail a pour orientation générale l'étude des conditions de vie et d'alimentation des abeilles dans le bassin méditerranéen.

Les recherches porteront tout d'abord sur les questions suivantes : désert;

1. Nectaires et secrétion nectarifère de différentes plantes et, en particulier, de plantes du désert;

2. Ressources polliniques offertes par différentes plantes et, en particulier, par les plantes du

3. Examen microscopique des miełs de différentes régions du bassin méditerranéen. Description des types de miels.

Il est prévu, dans le cours des prochaines années, d'organiser une réunion du groupe de travail "Flore méditerranéenne". 